\title{
Incômodo causado pelo ruído a uma população de bombeiros
}

\author{
Annoyance caused by noise to a population of firefighters
}

\author{
Millena Nóbrega Campos de Sousa ${ }^{1}$, Ana Cláudia Fiorini² ${ }^{2}$ Michelle Barna Guzman ${ }^{3}$
}

\begin{abstract}
RESUMO
Objetivo: Identificar o incômodo causado pelo ruído e queixas relacionadas à saúde auditiva em uma corporação de bombeiros do município Santo André (SP). Métodos: Tratou-se de um estudo epidemiológico transversal de inquérito na população de 72 bombeiros do $8^{\circ}$ Grupamento, 28 do setor administrativo, 20 do centro de ocorrência e 24 do atendimento operacional. Foi aplicado um protocolo composto por 57 questões referentes a dados pessoais, queixas auditivas, não auditivas e de incômodo. Também foi realizado o mapeamento sonoro do local. Resultados: Os níveis de pressão sonora no local da corporação ultrapassaram 67 dB (A), chegando a níveis maiores de $82 \mathrm{~dB}$ (A) na avenida. A maioria (83,3\%) dos sujeitos relatou o cotidiano ruidoso no trabalho, sendo o ruído urbano citado como maior fonte. Quando vistos separadamente por área de atuação, a maior ocorrência foi para ruído urbano $(73,9 \%)$, ruído da viatura $(68,0 \%)$ e ruído do telefone $(38,2 \%)$ para o setor administrativo, divisão operacional e atendimento de ocorrências, respectivamente. Conclusão: Os bombeiros analisados, além da exposição a ruídos provenientes da ocupação no ambiente de trabalho como viaturas, rádios de comunicação e telefones, também sofrem as consequências do ruído urbano, que altera atividades no trabalho e provoca incômodo.
\end{abstract}

Descritores: Audição; Ruído ocupacional; Zumbido/etiologia; Saúde do trabalhador

\section{INTRODUÇÃO}

O ruído representa um importante problema de saúde pública por estar presente em nosso cotidiano como, por exemplo, nos ambientes de trabalho, nas atividades de lazer e nas ruas das cidades. Tal fato tem contribuído para colocar a poluição sonora como a terceira maior causa de poluição no mundo, perdendo apenas para o ar e a água. Desta forma, o ruído pode representar um risco para a saúde de toda a população(1).

O ruído urbano é conhecido como poluição sonora e, apesar de representar um risco à saúde, ainda é um problema negligenciado pela população. Pode perturbar o trabalho, o descanso, o sono e a comunicação nos seres humanos; pode prejudicar a audição e causar ou provocar reações psicológicas, fisiológicas e até patológicas ${ }^{(2)}$.

O ruído não depende somente de suas características físicas, sendo importante observar o seu efeito em cada indivíduo

Extraído do trabalho realizado na Pontifícia Universidade Católica de São Paulo - PUC-SP - São Paulo (SP), Brasil, para obtenção do titulo de Mestre em Fonoaudiologia.

(1) Pós-graduanda (Mestrado) em Fonoaudiologia pela Pontifícia Universidade Católica de São Paulo - PUC-SP - São Paulo (SP), Brasil.

(2) Doutora, Professora Associada da Pontifícia Universidade Católica de São Paulo - PUC-SP - São Paulo (SP), Brasil.

(3) Mestre, Fonoaudióloga da Prefeitura Municipal de São Paulo - São Paulo (SP), Brasil.

Endereço para correspondência: Millena Nóbrega Campos de Sousa. R. Pageú, 48/173, Vila Mariana, São Paulo (SP), Brasil, CEP: 04139-000. E-mail: millenanobrega@yahoo.com.br

Recebido em: 28/7/2008; Aceito em: 12/4/2009 e em cada ambiente. Desta forma, o incômodo encontrado na população é de suma importância nos estudos de poluição sonora $^{(3)}$, apresentando-se como critério importante para avaliar os efeitos do ruído na saúde do indivíduo ${ }^{(4)}$.

Em 2004, a Organização Mundial de Saúde (OMS) $)^{(5)}$ ressaltou que, dentre os efeitos do ruído, a perda auditiva é o mais conhecido e pesquisado. Porém, o efeito na audição ocorre predominantemente nas exposições continuadas a elevados níveis de pressão sonora. Desta forma, a OMS enfatiza que os efeitos não auditivos merecem uma atenção especial, pois são decorrentes de exposições não tão elevadas como as que causam a perda auditiva. Como exemplo desses efeitos ressaltam principalmente o incômodo e as alterações no sono. O incômodo é descrito como uma sensação de desconforto decorrente do ruído ambiental e pode ser relatada pelos indivíduos como queixas de irritabilidade, estresse e mal-estar.

Componentes psicossociais da percepção do ruído foram avaliados em pesquisa que apontou o ruído urbano como maior fonte de incômodo. Os dados revelaram que, quanto maior a percepção negativa do ruído, maior é a percepção dos efeitos na saúde ${ }^{(6)}$. Os autores concluíram que existem diferentes fatores de ordem psicológica ou psicossocial que influem na percepção do ruído e nos efeitos percebidos nas exposições ${ }^{(7)}$.

O incômodo, denominado como um estresse psicológico ${ }^{(8)}$, pode estar associado tanto a fatores pessoais e sociais, quanto às características acústicas do ruído ${ }^{(9)}$. O nível da exposição não está diretamente vinculado à queixa, pois o incômodo pode ser relatado mesmo em situação não tão ruidosa ${ }^{(10)}$.

Estudos ressaltaram que as principais fontes de ruído 
urbano que podem causar incômodo na população são os veículos de transporte e os vizinhos, com frequência de $73 \%$ e 38\%, respectivamente. As queixas mais frequentes foram: irritabilidade, dificuldades de concentração, dor de cabeça, distúrbios de sono e alterações vestibulares ${ }^{(7,11-13)}$.

Diversos segmentos profissionais têm exposição a ruído em seus ambientes de trabalho, porém, é muito mais frequente observarmos estudos com trabalhadores de indústria e que abordam apenas os efeitos na audição. Outra profissão como, por exemplo, a de bombeiro, nem sempre é considerada exposta a ruído ocupacional. Alguns autores indicaram que esta profissão não proporciona risco para perda auditiva induzida por ruído ocupacional, pois, durante o trabalho, os deslocamentos não são padronizados e, dessa forma, a exposição a ruído ocorre ocasionalmente ${ }^{(14-16)}$. Entretanto, segundo outras pesquisas, os bombeiros ficam expostos a níveis de pressão sonora elevados, mesmo por períodos breves e intermitentes ${ }^{(17-18)}$. Além disso, o ruído urbano representa a exposição ocupacional para o bombeiro, uma vez que as corporações estão sempre localizadas em vias públicas de tráfego intenso de veículos.

Durante a jornada de trabalho dos bombeiros, algumas fontes mais específicas como, por exemplo, o ruído das sirenes dos veículos para locomoção, pode prejudicar severamente a inteligibilidade da fala. Alguns estudos apontaram que métodos para diminuir o ruído e realçar o sinal da fala durante uma situação de conversação podem ter resultados bastante satisfatórios $^{(19)}$.

Além do ruído, os bombeiros também ficam expostos a outros riscos ambientais e organizacionais. As exposições a ruído, produtos químicos, temperaturas elevadas, turnos rotativos de trabalho e tarefas executadas sob tensão, podem provocar diversos efeitos na saúde desta categoria profissional. Porém, dentre esses, destacamos o incômodo como um fator que pode alterar o estado de saúde geral e, consequentemente, diminuir a resistência do organismo diante das exposições a riscos durante a jornada de trabalho.

Programas de saúde e segurança ocupacional específicos para os bombeiros têm recebido uma atenção especial nos últimos anos, devido ao crescente reconhecimento do potencial de risco para a saúde desta população, a longo prazo. Além de enfrentarem severas exigências físicas e psicológicas, os bombeiros também estão expostos a riscos físicos (ruído e calor) e químicos (gases e solventes) que podem ocasionar consequências adversas ${ }^{(20)}$.

O ambiente de trabalho dos bombeiros é bastante diversificado e pode implicar vários riscos à saúde que vão desde leves ferimentos até mesmo à chance de óbito. Dentre as principais exposições podemos destacar os elevados níveis de ruído, as mudanças térmicas e a exposição a gases tóxicos. Vale ressaltar que o uso de equipamento de proteção individual nem sempre é freqüente, pois pode limitar a eficiência e o desempenho do trabalho ${ }^{(21)}$.

As atitudes e percepções dos bombeiros em relação à: audição, exposição ao ruído ocupacional, perda auditiva e ao uso de protetor também foram descritos em estudos qualitativos. Os autores ${ }^{(22)}$ evidenciaram que os bombeiros reconheceram a importância de uma boa audição no serviço de combate ao incêndio, porém, a perda auditiva foi considerada como um pequeno risco, quando comparada aos outros. Quanto ao uso de equipamento de proteção individual (EPI), acrescentaram que os bombeiros relataram que seu uso interfere na capacidade de ouvir os comandos durante situações de emergências. No entanto, reconheceram a importância da saúde auditiva e dos benefícios de um programa de conservação auditiva.

No Brasil, estudos sobre incômodo na população não são comuns e, quando ocorrem, frequentemente são executados por engenheiros que focam a atenção na avaliação quantitativa da poluição sonora. Na fonoaudiologia, estudos sobre o ruído e seus efeitos na saúde são realizados em populações de trabalhadores de indústrias e enfocam, portanto, as exposições ocupacionais. Porém, o ruído é um problema de saúde pública que atinge toda a população e, desta forma, os estudos sobre incômodo são importantes não somente para a avaliação qualitativa dos índices de poluição, mas principalmente, na geração de subsídios para as ações de educação ambiental.

Considerando os diversos riscos ambientais e de organização do trabalho aos quais a categoria profissional de bombeiros está submetida, estudos que identifiquem efeitos na saúde destes trabalhadores são necessários e podem gerar subsídios para o estabelecimento de ações de proteção com o objetivo de prevenção de doenças.

O objetivo desta pesquisa foi identificar queixas de saúde auditiva e o incômodo em relação ao ruído ocupacional e urbano, em uma corporação de bombeiros do município Santo André (SP).

\section{MÉTODOS}

Foi realizado um estudo epidemiológico do tipo transversal de inquérito na população de bombeiros do $8^{\circ}$ Grupamento e do Posto Campestre de Bombeiros do município de Santo André (SP), localizados na Avenida Prestes Maia, uma via de fluxo intenso de veículos de transporte. O estudo epidemiológico tranversal tem como principal característica avaliar situações de causa e efeito, simultaneamente.

Este estudo recebeu parecer favorável do Comitê de Ética em Pesquisa da Pontifícia Universidade Católica de São Paulo (PUC-SP) sob n ${ }^{\circ}$ 013/2005.

\section{Caracterização do local}

A sede da corporação abriga a administração da instituição que é responsável por 11 postos de bombeiros de sete cidades do Grande ABC (Santo André, São Bernardo, São Caetano, Diadema, Mauá, Ribeirão Pires e Rio Grande da Serra). O horário de funcionamento da sede é das $8: 30 \mathrm{~h}$ às $18: 15 \mathrm{~h}$, de segunda à sexta-feira. No local também funciona a Central de Operações do Bombeiro Militar (COBOM), que atende chamadas telefônicas de urgência (telefone 193) durante 24 horas com turnos rotativos de trabalho de 12 horas. O COBOM atende, em média, 1400 ligações diárias e estas geram aproximadamente 110 ocorrências. Em cada turno costumam trabalhar cinco profissionais no atendimento.

Anualmente, o número de ocorrências administradas pelo grupamento ultrapassa 20.000 registros, divididos entre salvamento, incêndio, resgate e trabalhos de auxilio à comunidade 
(TAC). Na sede, trabalham cerca de 60 funcionários com atividades administrativas.

\section{Casuística}

Participaram desta pesquisa todos os funcionários ativos no período de coleta. Após o primeiro contato, seguido da explanação acerca da pesquisa, todos os bombeiros aceitaram participar desta, constituindo uma amostra representada por todos os funcionários em atividade no período da coleta de dados. Foram avaliados 72 indivíduos sendo $95,8 \%$ do sexo masculino e 4,2\% do sexo feminino. Destes 72, 28 (38,9\%) eram do setor administrativo, $20(27,8 \%)$ do COBOM e 24 $(33,3 \%)$ do operacional. Os grupos foram denominados ADM, COBOM e OPER, respectivamente.

\section{Procedimentos de coleta}

Considerando que a pesquisa pretendeu avaliar o incômodo com relação ao ruído, foram necessárias algumas ações para constituir variáveis explicativas. Como não foi objetivo do estudo realizar avaliação audiométrica, a auto percepção da condição auditiva foi avaliada por meio de questionário, enfatizando a caraterística de estudo de inquérito. Além disso, foi realizado também o mapeamento sonoro da região do entorno do local sob estudo para reconhecer os níveis de pressão sonora que representam a exposição ocupacional.

\section{Mapeamento sonoro do local}

Foi utilizado o programa Sound Plan, que fornece um estudo detalhado envolvendo algumas áreas de tamanho e fontes múltiplas de ruído. Para a elaboração do mapa da presente pesquisa, o software foi alimentado com dados do mapa do município em versão "Autocad", além dos dados de contagem veicular da área estabelecida. A partir do fornecimento dos dados, o programa consegue estimar os níveis de pressão sonora do local e do entorno.

\section{Questionário}

Como instrumento de coleta de dados, foi utilizado um questionário adaptado a partir do instrumento elaborado ${ }^{(23)} \mathrm{na}$ execução do projeto "Poluição sonora na cidade de São Paulo", realizado em parceria da PUC-SP com a Secretaria do Verde e Meio Ambiente do município de São Paulo, no período de março de 2003 a julho de 2006.

A versão final do questionário, após as correções provenientes do estudo piloto do instrumento, foi composta por perguntas relativas a: dados pessoais, queixas auditivas, não auditivas, incômodo e sugestões referentes ao controle de ruído no local de trabalho.

\section{Procedimento para análise dos dados}

Após a análise de consistência do banco de dados, foram procedidos testes estatísticos. As análises foram realizadas no programa SPSS (Statistical Package for Social Sciences), versão 13.0. Inicialmente foi utilizado o Teste de KruskalWallis, com o intuito de verificar uma possível diferença entre os três grupos, quando comparados concomitantemente, para as variáveis de interesse. Considerando que algumas variáveis mostraram diferenças estatisticamente significantes entre os grupos, foi utilizado também o Teste de Mann-Whitney com o intuito de identificar quais grupos diferenciavam-se entre si, quando comparados par a par.

Foi adotado o nível de significância de 5\% $(0,05)$ para a aplicação dos testes estatísticos, ou seja, quando o valor da significância calculada (p) for menor do que 5\% $(0,05)$, há uma diferença dita estatisticamente significante, ou seja, uma diferença efetiva. Quando o valor da significância calculada (p) for igual ou maior do que $5 \%(0,05)$ a diferença é considerada estatisticamente não significante, ou seja, pode implicar apenas uma semelhança.

\section{RESULTADOS}

\section{Resultados do mapeamento do ruído}

O mapeamento dos níveis sonoros nas proximidades da Avenida Prestes Maia, onde está localizado o $8^{\circ}$ Grupamento e o Posto Campestre de Bombeiros do município de Santo André, indicou níveis médios de 76 a 82 dB (A). Nas regiões no entorno foram obtidos níveis de 67 a $73 \mathrm{~dB}$ (A). Portanto, o local de trabalho do Grupamento apresentou níveis superiores a $73 \mathrm{~dB}(\mathrm{~A})$.

\section{Resultados do protocolo de avaliação}

Dos 72 sujeitos, a média do tempo de trabalho na administração (grupo ADM) foi de 14,36 anos ( $\mathrm{DP}=7,15)$, no COBOM (grupo COBOM) foi 15,7 anos (DP=5,44) e no Operacional (grupo OPER) foi 14,62 anos de prestação de serviço $(\mathrm{DP}=5,66)$. Em relação ao tempo na função de bombeiro, foi observada uma diferença estatisticamente significante, indicando a maior média de tempo na função para os bombeiros da divisão operacional (140,7 meses). A administração teve como média de 71,1 meses e o COBOM de 77,8 meses. A queixa de presença de ruído no local de trabalho foi relatada por 60 sujeitos $(83,3 \%)$. A distribuição, por grupo, foi de $75 \%$ para o ADM, $85 \%$ para o COBOM e $91,7 \%$ no Operacional (OPER). Do total, a maioria relatou que o ambiente é sempre ruidoso $(81 \%, 82,4 \%$ e $72,7 \%$, respectivamente).

A partir da constatação de presença de ruído no local de trabalho, a etapa seguinte foi investigar as principais fontes de poluição sonora relatada pelos três grupos. A distribuição das fontes sonora variou de acordo com o setor de atuação profissional, como pode ser observado na Tabela 1. A aplicação do teste de Kruskal-Wallis identificou diferenças estatisticamente significantes entre os grupos $(\mathrm{p}<0,001)$. Em seguida, o teste de MannWhitney possibilitou identificar quais grupos diferenciam-se entre si, quando comparados par a par. De uma maneira geral, o grupo OPER é o que mais se diferencia dos demais, no que diz respeito à citação de fontes de ruído no local de trabalho. A comparação do Grupo OPER com os grupos ADM e COBOM identificou $\mathrm{p}=0,001$ e $\mathrm{p}=0,002$, respectivamente. 
Apesar do ruído da avenida ser citado em todos os grupos, foi mais frequente apenas para o grupo $\operatorname{ADM}(73,9 \%)$. Para a central de atendimento (grupo COBOM) o maior número de reclamações $(38,2 \%)$ foi do som do telefone, seguido do rádio de comunicação $(23,5 \%)$ e avenida $(23,5 \%)$. Já para a divisão operacional, a maioria $(68,0 \%)$ indicou a viatura como principal fonte de ruído, seguido do ruído da avenida $(28,0 \%)$.

Tabela 1. Distribuição das fontes de ruído no local de trabalho $(\mathrm{N}=60)$

\begin{tabular}{lcccc}
\hline Fonte & \multicolumn{3}{c}{ Setor } & \multirow{2}{*}{ Total } \\
\cline { 2 - 4 } & ADM & COBOM & OPER & \\
& $\mathrm{N}(\%)$ & $\mathrm{N}(\%)$ & $\mathrm{N}(\%)$ \\
\hline Avenida & $17(73,9)$ & $8(23,5)$ & $14(28,0)$ & $39(36,5)$ \\
Telefone & $2(8,7)$ & $13(38,2)$ & $-(0,0)$ & $15(14,0)$ \\
Pessoas & $1(4,4)$ & $5(14,7)$ & $-(0,0)$ & $6(2,8)$ \\
Equipamentos & $3(13,0)$ & $-(0,0)$ & $-(0,0)$ & $3(2,8)$ \\
da sala & & & & \\
Rádio & $0(0,0)$ & $8(23,5)$ & $2(4,0)$ & $10(9,4)$ \\
Viatura & $-(0,0)$ & $-(0,0)$ & $34(68,0)$ & $34(31,8)$ \\
\hline
\end{tabular}

Legenda: $\mathrm{ADM}=$ administrativo; $\mathrm{COBOM}=$ central de operações do bombeiro militar; OPER = operacional

A Figura 1 evidencia que a maioria dos sujeitos $(78,3 \%)$ da esfera administrativa (grupo ADM) relatou que o ruído no local de trabalho altera a concentração. No setor de atendimento telefônico de ocorrência (grupo COBOM) a maior alteração que o ruído causa para a maioria é dificuldade para ouvir $(58,3 \%)$, seguida de problemas de concentração $(16,7 \%)$. Já para a divisão operacional (grupo OPER) as mais citadas foram situação crítica para dormir $(22,2 \%)$, seguida de dificuldade para ouvir $(16,7 \%)$.

Os dados da Tabela 2 também revelaram diferenças estatisticamente significantes entre os grupos analisados. O teste de Kruskal-Wallis identificou diferenças estatisticamente significantes entre os grupos $(\mathrm{p}<0,013)$. Em seguida, o teste de Mann-Whitney identificou que o grupo COBOM, que não apresentou nenhuma queixa de audição, se diferencia dos demais grupos (ADM e OPER) com significância de $\mathrm{p}=0,003$ e $\mathrm{p}=0,009$, respectivamente.
Tabela 2. Distribuição da população pesquisada quanto a problemas de audição que necessitaram de cuidados médicos ( $\mathrm{N}=72)$

\begin{tabular}{lcccc}
\hline Problemas de & \multicolumn{4}{c}{ Setor } \\
audição & ADM & COBOM & OPER \\
& $\mathrm{N}(\%)$ & $\mathrm{N}(\%)$ & $\mathrm{N}(\%)$ \\
\hline Sim & $10(35,7)$ & $-(0)$ & $7(29,2)$ & $17(23,6)$ \\
Não & $18(64,3)$ & $20(100,0)$ & $17(70,8)$ & $55(76,4)$ \\
\hline
\end{tabular}

Legenda: $A D M=$ administrativo; $C O B O M=$ central de operações do bombeiro militar; OPER = operacional

Quanto à realização de exames de audição dos 72 participantes da pesquisa, $25(34,7 \%)$ afirmaram ter realizado exames de audição devido aos seguintes fatores: 19 (76\%) por sensação de ouvidos tampados, três $(12 \%)$ por traumas (barotrauma e traumatismo craniano), dois (8\%) por outras doenças (caxumba e labirintite) e um sujeito (4\%) por necessidade em trabalhos anteriores. Os exames realizados nesta população foram, em maioria, a audiometria $(88,5 \%)$, seguida de exame otoneurológico (3,9\%). Os demais $(3,9 \%)$ não lembraram a quais procedimentos foram submetidos. Dos 25 bombeiros que realizaram exames auditivos, $20(76,9 \%)$ relataram resultados normais, quatro $(15,4 \%)$ alteração leve e um sujeito $(3,9 \%)$ não lembrou do resultado. A divisão operacional se diferenciou das demais, tendo em sua maioria $(58,3 \%)$ a resposta afirmativa para a realização de exame de audição anterior.

Os participantes da pesquisa foram questionados sobre a percepção da audição, no momento da realização do questionário e a maioria $(55,6 \%)$ relatou ter audição boa, seguida de ótima e regular.

A Figura 2 mostra as queixas decorrentes do ruído no local de trabalho por ordem de ocorrência, a saber: incômodo $(25,6 \%)$, intolerância e irritabilidade $(20,8 \%)$, dor de cabeça $(14,5 \%)$, diminuição da audição $(14,0 \%)$, alteração do sono $(13,5 \%)$, zumbido $(10,1 \%)$ e tontura $(1,5 \%)$.

Com relação à queixa de tontura, independentemente da relação com o ruído no local de trabalho, 9,7\% do total dos três grupos responderam afirmativamente. A Figura 3 apresenta a ocorrência da queixa de tontura, por grupo. Dentre os participantes que responderam sim para queixa de tontura, $85,7 \%$ relataram a frequência às vezes e $14,3 \%$ sempre.

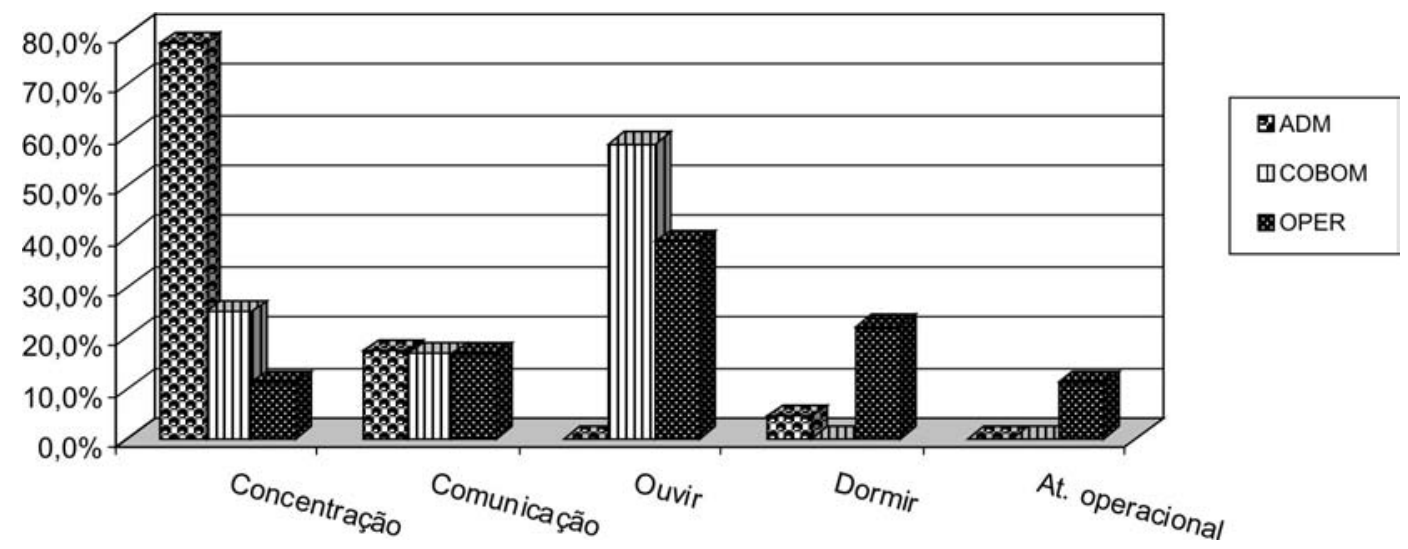

Figura 1. Distribuição da população analisada quanto ao tipo de interferência decorrente de ruído durante as atividades laborativas ( $\mathrm{N}=38$ ) Legenda: At. operacional $=$ atendimento operacional 


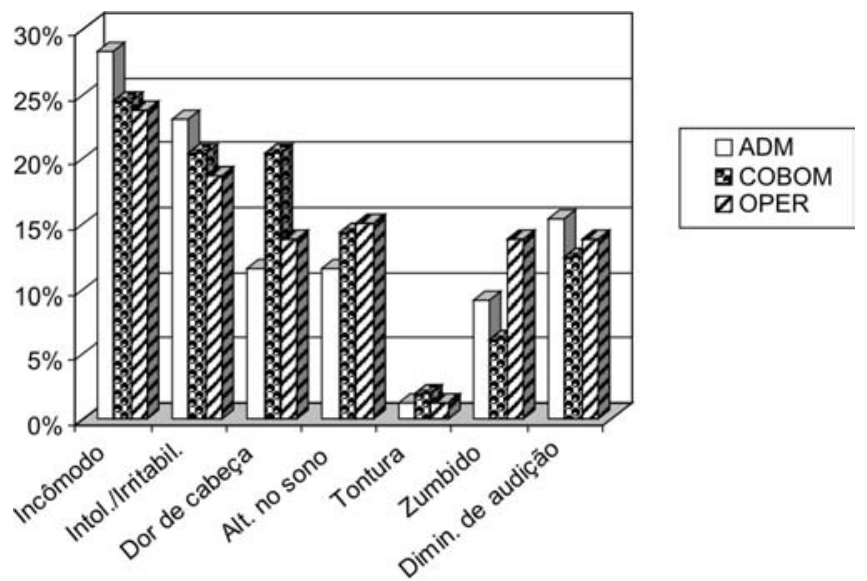

Figura 2. Distribuição de queixas decorrentes ao ruído presente no local de trabalho $(\mathrm{N}=72)$

Legenda: Intol./Irritabil. = intolerância/irritabilidade; Alt. no sono = alteração no sono; Dimin. de audição = diminuição de audição

A Figura 4 apresenta a ocorrência da queixa de zumbido por grupo. Dentre os participantes que responderam sim para queixa de zumbido, $90 \%$ apresentam às vezes e $10 \%$ sempre. A classificação do tipo de zumbido indicou maior ocorrência para barulho de apito (68\%), seguida de abelha (8\%) e de chuva (4\%).

\section{DISCUSSÃO}

Nos âmbitos federal, estadual e municipal existem diversas leis e resoluções referentes ao ruído como risco físico. $\mathrm{O}$ Ministério do Trabalho e Emprego, a Associação Brasileira de Normas Técnicas, o Conselho Nacional do Meio Ambiente e o próprio município de Santo André ditam limites máximos, padrão de emissão e controle da poluição sonora, condições para avaliação de ruído e critérios de avaliação. Considerando a condição de conforto acústico, os níveis obtidos nesta pesquisa estão acima dos recomendados pela legislação vigente. Os resultados corroboram estudos cujos nivéis de ruído urbano identificados em várias cidades também excederam aos valores permitidos por lei $i^{(3,24)}$.

A análise separada por grupos (Tabela 1) indicou que o ruído urbano aparece na segunda opção para o COBOM e atendimento operacional (OPER). Tais grupos se preocuparam inicialmente com fontes como telefones e viaturas, respectivamente. O que parece ocorrer é que a fonte apontada por esses setores mascara o ruído considerado de fundo (ruído urbano), não permitindo que mesmo seja apontado como fonte principal, mas sim, como secundária. A percepção do ruído depende da atividade que está sendo desenvolvida e, desta forma, a demanda ocupacional pode estar relacionada ao tipo de fonte que mais atrapalha o desempenho na função ${ }^{(7)}$.

As principais interferências nas atividades laborativas decorrentes do ruído no ambiente de trabalho foram: dificuldade de concentração $(43,4 \%)$, ouvir $(26,4 \%)$ e alteração na comunicação $(16,9 \%)$. Pesquisadores também observaram que as queixas de irritabilidade e baixa concentração foram as de maior ocorrência em estudos sobre os principais efeitos do ruído urbano na saúde ${ }^{(12)}$. Considerando que os níveis de ruído obtidos no mapeamento sonoro desta pesquisa apontaram valores superiores a $67 \mathrm{~dB}(\mathrm{~A})$, era de se esperar que as queixas de alterações na concentração e dificuldades para ouvir e se comunicar no local de trabalho estivessem presentes na população de bombeiros.

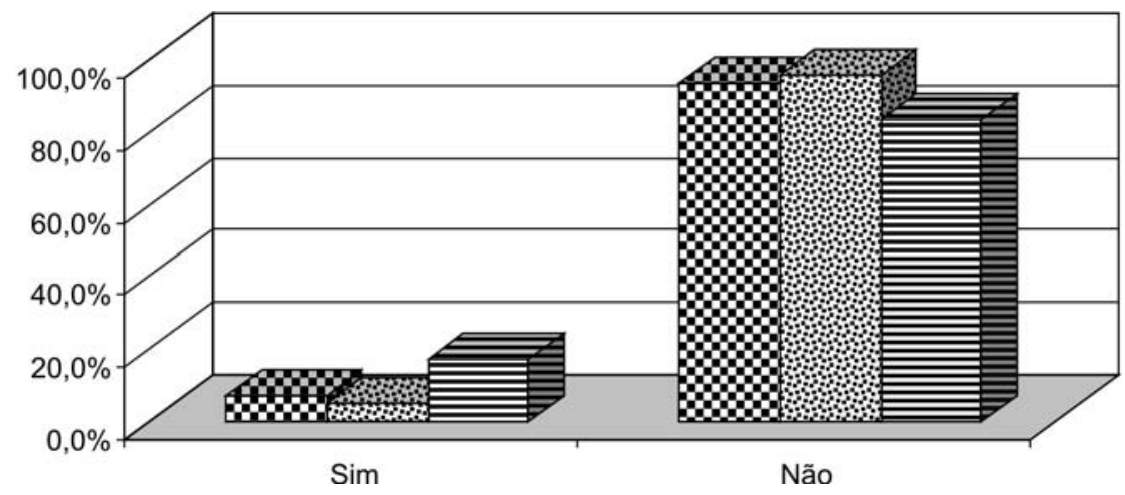

ه ADM
बCOBOM
日OPER

Figura 3. Distribuição de queixa de tontura na população analisada $(\mathrm{N}=72)$
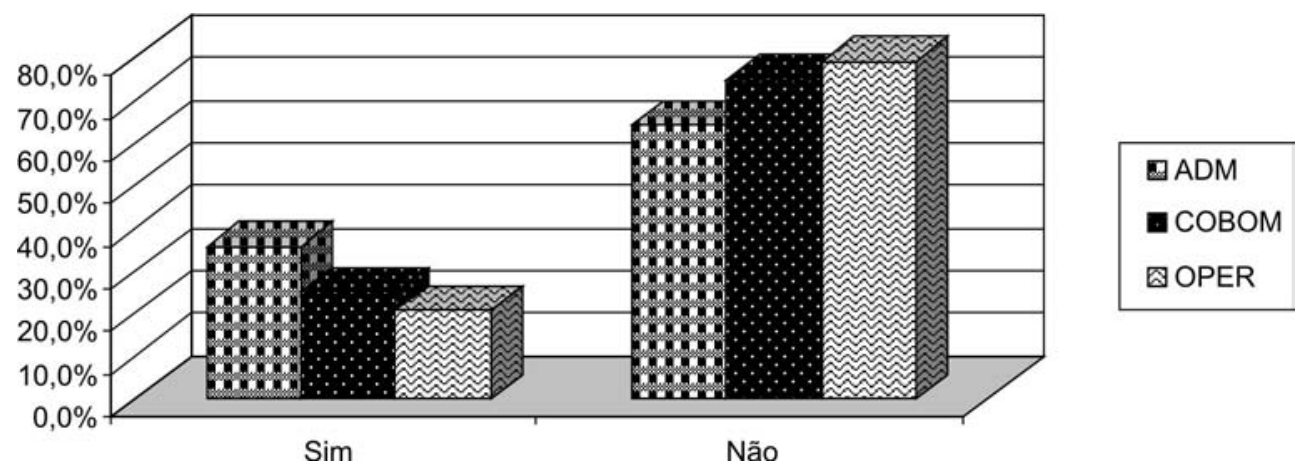

Figura 4. Distribuição da população pesquisada quanto à presença de zumbido $(\mathrm{N}=72)$ 
Com relação às queixas decorrentes do ruído no local de trabalho, o incômodo foi relatado em $(25,6 \%)$, seguido de intolerância e irritabilidade $(20,8 \%)$, dor de cabeça $(14,5 \%)$, diminuição da audição $(14,0 \%)$, alteração do sono $(13,5 \%)$, zumbido $(10,1 \%)$ e tontura $(1,5 \%)$, respectivamente. Tais resultados vão ao encontro dos estudos que observaram as mesmas queixas decorrentes principalmente do ruído urbano ${ }^{(3,6,8-10)}$. Pesquisas também relataram que o ruído ambiental gera como principais queixas: irritabilidade (58\%), baixa de concentração (42\%), insônia (20\%) e dores de cabeça $(20 \%)^{(11)}$.

A queixa de tontura $(9,7 \%)$ foi inferior à de zumbido $(27,8 \%)$, porém, os que apresentaram tais queixas indicaram, em maioria, a frequência às vezes $(85,7 \%$ e $90 \%$, respectivamente). É reconhecido que a exposição crônica ao ruído pode acarretar alterações vestibulares. Os presentes dados corroboram outro estudo que identificou tendência de correlação entre grau de perda auditiva e prevalência de distúrbios vestibulares. A referida pesquisa identificou a ocorrência de distúrbios vestibulares observada nos pacientes por meio de queixas de tontura e zumbido ${ }^{(13)}$.

Para os alguns autores ${ }^{(16,19)}$, a profissão de bombeiros oferece vários perigos à saúde, dentre eles, os efeitos da exposição a ruído. Assim, medidas de proteção coletiva devem ser adotadas com o objetivo de minimizar a ocorrência de alterações de saúde que podem prejudicar a qualidade de vida dos bombeiros.

\section{CONCLUSÃO}

Considerando todos os resultados obtidos nesta pesquisa, pode-se afirmar que, para os bombeiros, o ruído urbano representa a sua exposição ocupacional. Além disso, outros riscos estão presentes nos ambientes de trabalho desta categoria profissional e podem, na exposição simultânea com o ruído, aumentar a chance de efeitos na saúde.

Foi possível observar também uma alta ocorrência de queixas de saúde e de alterações durante a execução das tarefas, relacionadas à exposição a ruído no local de trabalho. O incômodo em relação ao ruído no local de trabalho foi a queixa de maior ocorrência.

A partir dos resultados desta pesquisa, fica evidente a importância de outros estudos que possam avaliar de forma mais específica todos estes efeitos observados na saúde dos bombeiros. Desta forma, torna-se fundamental a implementação de medidas de proteção coletiva e individual com o objetivo de prevenir efeitos deletérios à saúde desta categoria profissional.

\section{AGRADECIMENTOS}

À Coordenação de Aperfeiçoamento Pessoal de Nível Superior (CAPES) pelo fomento concedido, imprescindível para a realização deste trabalho.

\begin{abstract}
Purpose: To identify annoyance caused by occupational and urban noises and auditory health complaints of a group of firefighters from a corporation in Santo André (SP), Brazil. Methods: A cross-sectional epidemiological study was carried out with a population of 72 firefighters from the $8^{\text {th }}$ Brigade: 28 firefighters from the administrative section, 20 from the occurrence center and 24 from the operational call center. It was used a protocol which consisted of 57 questions regarding personal data, auditory and non-auditory complaints and annoyance. A sound mapping from the surrounding area was also carried out. Results: The sound pressure levels in the firefighter corporation area exceeded $67 \mathrm{~dB}(\mathrm{~A})$, reaching more than $82 \mathrm{~dB}(\mathrm{~A})$ at the main avenue. Most of the subjects (83.3\%) reported noisy routine at work, and urban noise was reported as the major source of annoyance. When evaluated separately by performance section, the highest occurrences of complaints were for urban noise (73.9\%), firefighter vehicle noise (68\%) and telephone noise (38.2\%), for the administrative section, operational call center and occurrence center, respectively. Conclusion: The firefighters interviewed, besides being exposed to noises deriving from work activities, like vehicles, walk-talk radios and telephones, are liable to consequences from urban noise, which disturbs work activities and also causes health problems.
\end{abstract}

Keywords: Hearing; Noise, occupational; Tinnitus/etiology; Occupational, health

\title{
REFERÊNCIAS
}

1. Fiorini AC. Ruído: um problema de saúde pública. Quebrando o Silêncio. 1997 jul/ago:3-4.

2. Organização Panamericana de la Salud. Organização Mundial de la Salud. Critérios de salud ambiental - 12: el ruído. México: OPAS; 1980

3. Martín MA, Tarrero A, González J, Machimbarrena M. Exposure-effect relationships between road traffic noise annoyance and noise cost valuations in Valladolid, Spain. Appl Acoust. 2006;67(10):945-58.

4. Wanner HU. [Annoyance due to street traffic and aircraft noise]. Soz Praventivmed. 1982;27(2-3):119-23. German.
5. WHO (World Health Organization). 2004. WHO LARES Final Report Noise effects and morbidity. In: Niemann H, Maschke C. Interdisciplinary research network "Noise and Health. Berlin Center Public Health; 2004. p. 1-20.

6. Azevedo R, Lima ML. Componentes psicossociais do ruído: as medições cognitivas do ruído em diferentes grupos profissionais. In: $1^{\circ}$ Colóquio psicologia espaço e ambiente. Universidade de Évora 2002; maio 9-10: $1-11$. 
7. Gouveia VV, Günther H. Percepção de incômodo ao barulho ambiental: um modelo parcial e preliminar. Estud Psicol (Campinas). 1996;13(2):17-28.

8. Stallen PJ. A theoretical framework for environmental noise annoyance. Noise Health. 1999;1(3):69-80.

9. Flindell IH, Stallen PJ. Non-acoustical factors in environmental noise. Noise Health. 1999;1(3):11-6.

10. Job RF. Noise sensitivity as a factor influencing human reaction to noise. Noise Health. 1999;1(3):57-68.

11. Zannin PHT, Calixto A, Diniz FB, Ferreira JA, Schuhli RB. Incômodo causado pelo ruído urbano à população de Curitiba, PR. Rev Saúde Pública = J Public Health. 2002;36(4):521-4.

12. Paz EC, Ferreira AMC, Zannin PHT. Estudo comparativo da percepção do ruído urbano. Rev Saúde Pública = J Public Health. 2005;39(3):46772.

13. Castro JC. Da avaliação da função vestibular em pacientes com perda auditiva induzida pelo ruído: pesquisa à vectoeletronistagmografia. [tese]. São Paulo: Universidade Federal de São Paulo. Escola Paulista de Medicina; 2000.

14. Reischl U, Bair HS Jr, Reischl P. Fire fighter noise exposure. Am Ind Hyg Assoc J. 1979;40(6):482-9.

15. Reischl U, Hanks TG, Reischl P. Occupation related Fire fighter hearing loss. Am Ind Hyg Assoc J. 1981;42(9):656-62.

16. Ewingman BG, Kivlahan CH, Hosokawa MC, Horman D. Efficacy of an intervention to promote use of hearing protection devices by firefighters. Public Health Rep. 1990;105(1):53-9.
17. Kales SN, Freyman RL, Hill JM, Polyhronopoulos GN, Aldrich JM, Christiani DC. Firefighters' hearing: a comparison with population databases from the International Standards Organization. J Occup Environ Med. 2001;43(7):650-6.

18. Clark WW, Bohl CD. Hearing levels of firefighters: risk of occupational noise-induced hearing loss assessed by cross-sectional and longitudinal data. Ear Hear. 2005;26(3):327-40. Comment in: Ear Hear. 2008;29(5):815-6; author reply 816-7; discussion 817-8.

19. Ding H, Lu J, Qiu X, Xu B. An adaptive speech enhancement method for siren noise cancellation. Appl Acoust. 2004;65(4):385-99.

20. Melius J. Occupational health for firefighters. Occup Med. 2001;16(1):101-8.

21. Matticks LA, Westwater JJ, Himel HN, Morgan RF, Edlich RF. Health risks to fire fighters. J Burn Care Rehabil. 1992;13(2 Pt 1):223-35.

22. Hong O, Samo D, Hulea R, Eakin B. Perception and attitudes of firefighters on noise exposure and hearing loss. J Occup Environ Hyg. 2008;5(3):210-5.

23. Fiorini AC, Fratti RRA, Oliveira BP, editores. Poluição sonora na cidade de São Paulo: estudo dos níveis de pressão sonora e o incômodo da população residente no entorno do Parque do Ibirapuera. In: I SIMBRASE - Simpósio de Acústica de Salas, Edificações e Escolas. Anais. São Paulo; 2006. p. 32-3.

24. Rossi MM. O impacto do ruído urbano sobre a audição: estudo comparativo entre operadores de tráfego da CET e guardas florestais. [dissertação]. São Paulo: Faculdade de Medicina da Universidade de São Paulo; 2002. 